Artículo

\title{
Escuela y violencia: estudio de caso del Instituto Nacional de Santa Lucía
}

\author{
School and violence: the case study of Instituto Nacional \\ de Santa Lucía
}

Roberto Alexander Damas Solórzano*

roberto.damas@udb.edu.sv

ISSN 1996-1642, Editorial Universidad Don Bosco, año 10, No.17, enero-junio de 2016, pp. 11-25 Recibido: 29 junio de 2015. Aprobado: 11 de enero de 2016

\begin{abstract}
Resumen
Este artículo presenta los resultados del estudio de caso en el Instituto Nacional de Santa Lucía, ubicado en el Municipio de llopango (El Salvador), donde se estudió la convivencia de la Comunidad Educativa de dicha institución y su relación con la violencia. Los resultados ilustran que el contexto actual tiende a dar exclusividad a la violencia pandilleril, invisibilizando otras manifestaciones de violencia de tipo directa, estructural y cultural. Los resultados de la investigación evidencian la existencia de otras concreciones de violencia que desde la teoría de Joseph Galtung, no son tomadas en cuenta para las diferentes intervenciones de prevención, planes o estrategias institucionales de prevención.
\end{abstract}

\begin{abstract}
This paper shows the results of the case study carried out at Instituto Nacional de Santa Lucía, located in Ilopango, San Salvador (El Salvador) where the core study was coexistence of members of the school community in relation to violence. Results show that current nationwide gangbased violence context hides other forms of violence such as direct, structural and cultural violence. The outcomes of this research also show other materializing ways that, under Johan Galtung's theory, are neglected when prevention interventions and plans are designed and implemented.
\end{abstract}

Keywords: direct violence, gender violence, student, educational community.

Palabras clave: Violencia directa, violencia de género, estudiantes, comunidad educativa.

\footnotetext{
* El autor es Maestro en Políticas para la prevención de violencia juvenil en cultura de paz, Director de Pastoral Universitaria y catedrático de la Universidad Don Bosco.
}

Para citar este artículo: Damas, R. A. (2016). Escuela y violencia: estudio de caso del Instituto Nacional de Santa Lucía. Diá-logos 17, 11-25. 


\section{Introducción}

La educación es un derecho inherente a la persona humana. En el contexto actual de El Salvador, el ejercicio de este derecho a la educación se ve afectado no solo por las condiciones de pobreza, desigualdad y desempleo, sino también por la violencia social que incide negativamente en la consecución efectiva de los fines de la educación, especialmente en las niñas, niños, adolescentes y jóvenes que asisten a centros escolares públicos.

Con respecto a eso, el informe del PNUD señala:

Se convierte en un desafío el educar en un entorno donde la inseguridad es la norma, y donde la violencia modifica y afecta no solo los diferentes tipos de relaciones -entre iguales, con docentes, entre amigos y con desconocidos, sino también en las conductas y los incentivos. La función de la escuela pública se muta, se altera y en vez de constituirse en un espacio de protección se convierte en uno de riesgo, del cual algunos deciden alejarse, a veces de manera temporal y otras, definitiva... (PNUD 2., 2014, p. 223)

Según los datos de FLACSO (2013) en El Salvador, durante 2011 fueron asesinadas 6 personas con profesión docente, 140 niños/niñas y/o jóvenes que eran alumnos del sistema educativo nacional. Para el 2012 los asesinatos fueron 57, lo que representó una reducción de casi el 60\% de casos comparado con el 2011 . De los 57 asesinados 21 estudiaban Bachillerato; 27 Tercer ciclo y 9 eran de algún grado de primero-segundo ciclo de educación básica.

Las autoridades del Ministerio de Educación han desarrollado diferentes estrategias para disminuir y controlar el impacto de la violencia que afecta a las escuelas. Dentro de estas estrategias está el Plan de Prevención y Seguridad Escolar 20132014 (PLANPREVES), que busca articular a través de una comisión interinstitucional la prevención de la violencia escolar para impulsar acciones que contribuyan a mejorar la seguridad y cultura de convivencia en el entorno educativo.

En el diagnóstico del PLANPREVES se cita que en los cuatro últimos años, la violencia en torno a las escuelas cobró la vida de 286 niños, jóvenes y docentes en el país. El Ministerio de Educación plantea que estas muertes no son causadas por "la violencia escolar"3 sino que han ocurrido dentro de las categorías aquí llamadas como "violencia contextual" o "violencia en la escuela"; es decir, están más relacionadas con las prácticas delincuenciales y del crimen organizado que asedian a las instituciones escolares.

Escuela y violencia: estudio de caso del Instituto Nacional de

12. Santa Lucía
3. Como parte de lo que se considera violencia escolar lo más relevante que se destaca es el castigo físico y psicológico, especificando conductas de maltrato de docentes a alumnos, hay acoso, exclusión, maltrato y hostigamiento entre alumnos, robos, hurtos, peleas y amenazas entre alumnos, asimismo, violencia sexual y de género, donde se evidencia conductas como acoso sexual entre alumnos, de docentes a estudiantes, discriminación por preferencia sexual entre alumnos. (PLANPREVES, 2013) 
En el año 2010, el Ministerio de Educación en coordinación con la organización no gubernamental Plan Internacional El Salvador, inauguraron una campaña "aprender sin miedo". Sin embargo, en una encuesta realizada por PLAN revelaba que el $42 \%$ de los entrevistados confirmó haber sufrido violencia psicológica y agresión de sus padres y docentes; un $25 \%$ dijo que sufrió violencia física de sus compañeros en los últimos tres meses y el 10\% de las alumnas reveló que había sido víctima de acoso o abuso sexual (Lemus, 2010).

En el contexto y la diferencia que el PLANPREVES 2013 determina entre violencia escolar y violencia contextual, motivó el estudio de caso en el Instituto Nacional Santa Lucía (INSL). El propósito de dicho estudio era analizar las concreciones de violencia desde la perspectiva de la convivencia que experimentan específicamente los miembros de la comunidad educativa.

El estudio se realizó en dicho centro educativo por pertenecer al sistema público. Este municipio se ubicaba para el año 2011 en la posición número 11 entre los 20 municipios con mayor porcentaje de hurto, robos, extorsiones y homicidios (FUNDAUNGO, 2012). En ese contexto se buscó analizar como incidía la violencia en el ámbito escolar y sus concreciones.

\section{Marco teórico: La teoría del conflicto de Johan Galtung}

Aunque existen muchos enfoques que permiten abordar la paz, la violencia y el conflicto, el presente estudio toma como referente teórico la teoría del conflicto de Johan Galtung. La teoría de Galtung está presente en el 50\% de estudios para la paz, y continua siendo un punto teórico de referencia para casi todos los investigadores en el campo de los estudios para la paz y la violencia (Calderón, 2009). Es este enfoque teórico el que ha permitido categorizar las diferentes concreciones de violencia a lo largo de 50 años y de la cual han partido muchos investigadores para crear sus teorías sobre la violencia y la paz.

En Galtung está claro el principio: una teoría de conflictos, no solo debe reconocer si los conflictos son buenos o malos; esta deberá fundamentalmente ofrecer mecanismos para entenderlos lógicamente, criterios científicos para analizarlos así como metodologías (creatividad, empatía y no violencia) para transformarlos.

La teoría del conflicto no busca enfrascar los conflictos en buenos y malos, se fundamenta en ejercer mecanismos para entenderlos lógicamente, criterios científicos para analizarlos, así como metodologías (creatividad, empatía y no violencia) para transformarlos, es decir, "un conflicto no se soluciona, se transforma" (Galtung, 2006).

Estos estudios se originan a raíz de la guerra fría, los cuales se basan en una visión holística del conflicto:

- Micro: Personas

- Meso: grupo (relacional) 
- Macro: sociedad, Estado y nación

- Mega: región, civilización

El conflicto para Galtung se puede observar mediante un triángulo:

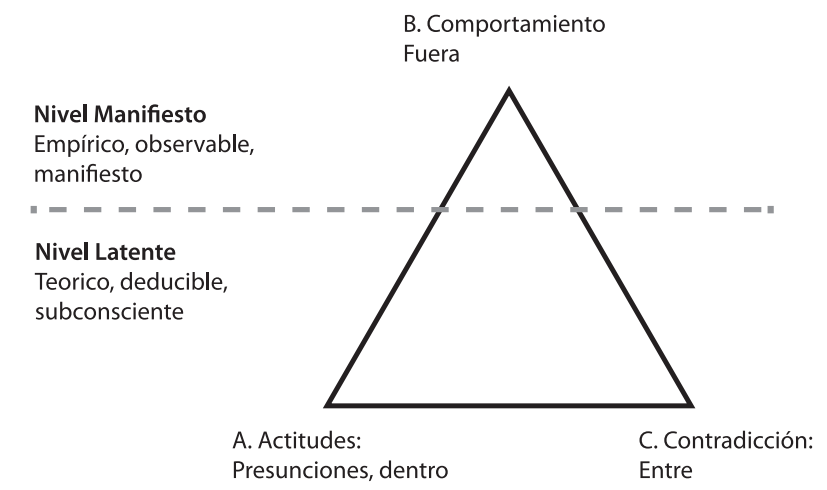

Las actitudes son el aspecto motivacional: cómo se sienten, cómo piensan las partes en el conflicto, como perciben al otro. El comportamiento (aspecto objetivo) alude al actuar de las partes en conflicto. La contradicción (aspecto subjetivo) tiene que ver con el tema o temas reales del conflicto y cómo se manifiestan.

Asimismo, los conflictos pueden tener un nivel observable o manifiesto y un nivel latente. Normalmente el aspecto manifiesto de un conflicto es el comportamiento $\mathrm{B}$, mientras que $\mathrm{A}$ y $\mathrm{C}$ permanecerán en el nivel latente. Por otro lado, un conflicto puede iniciarse en cualquiera de los ángulos. El no percibir el conflicto sin estas tres dimensiones resulta en una percepción limitada del mismo, por lo que puede llevar a un manejo inadecuado del conflicto.

Los problemas más comunes en la prevención al comprender inadecuadamente el conflicto son:

Focalizar la atención solo en las actitudes lleva a sostener que los problemas derivan de mentes tomadas por el odio o de mentes distorsionadas, quizás necesitadas de conversión religiosa/ideológica, de psicoterapia y/o de educación a la paz y al conflicto, permaneciendo ciegos/as frente al hecho que también la mente más normal puede asesinar o tolerar un asesinato cuando las contradicciones que lo frustran se prolongan en el tiempo.

Focalizar la atención sólo sobre el comportamiento es muy frecuente dado que ahí es donde reside la violencia. Someter las personas puede servir para barrer debajo de la alfombra de la violencia, el problema, haciéndola menos evidente. Pero no influirá positivamente en la contradicción subyacente.

Escuela y violencia: estudio de caso del Instituto Nacional de 14. Santa Lucía
Focalizar la atención sólo sobre la contradicción basándose en la ingeniería social, corre el riesgo de intensificar el odio y la violencia, si la aproximación a la contradicción es violenta. 
Por ello la visión holística del conflicto, debe incluir estas dimensiones, los átomos del conflicto:

- Disputa: dos personas que persiguen un mismo fin que escasea (entre las personas)

- Dilema: Una persona, actor, que persigue dos fines incompatibles entre sí. (dentro de las personas).

Desde estos átomos del conflicto se pueden analizar estructuras conflictuales más complejas.

Por ello el conflicto que no es transformado desencadena en violencia, que para Galtung tiene una triple dimensión: Directa, Estructural y Cultural.

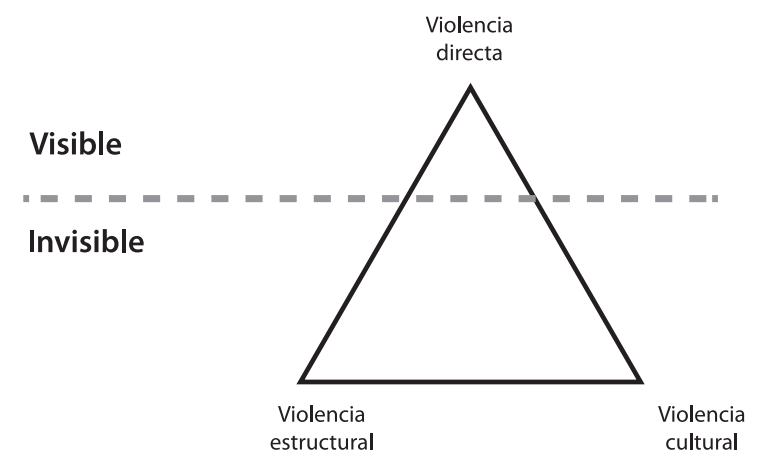

La violencia directa es la violencia manifiesta, es el aspecto más evidente de esta. Su manifestación puede ser por lo general física, verbal o psicológica. La violencia estructural se trata de la violencia intrínseca a los sistemas sociales, políticos y económicos mismos que gobiernan las sociedades, los estados y el mundo. Su relación con la violencia directa es proporcional a la parte del iceberg que se encuentra sumergida en el agua. La violencia cultural son aquellos aspectos de la cultura, en el ámbito simbólico de nuestra experiencia (materializado en la religión e ideología, lengua y arte, ciencias empíricas y ciencias formales, símbolos: cruces, medallas, medias lunas, banderas, himnos, desfiles militares, etc.), que puede utilizarse para justificar o legitimar la violencia directa o estructural (Galtung, 2003). Sería la suma total de todos los mitos: de gloria y trauma y demás, que sirven para justificar la violencia directa (Galtung, 1998). La gran complejidad de los problemas, peligros y oportunidades que emergen de la violencia en general y de la guerra en particular requiere de respuestas igualmente complejas y multidimensionales. Galtung propone la reconstrucción (tras la violencia directa), reconciliación (de las partes en conflicto) y más que nunca, resolución (del conflicto subyacente) (Galtung, 1998).

La transformación del conflicto y violencia que plantea Galtung, es la perspectiva que consideramos estrechamente vinculada a los presupuestos de los Estudios para la paz. Como dirá también Vicent Fisas (1998), refiriéndose a la transformación. Esta aprovecha el conflicto para construir la paz a través de compromisos a largo plazo que involucran toda la sociedad. Desde esta forma 
de intervenir, los conflictos se convierten en retos que brindan la posibilidad de crear oportunidades positivas.

\section{Método}

Se realizó un estudio de caso (Martínez, 2006) en el Instituto Nacional Santa Lucia, a través del método cualitativo con un enfoque integral del abordaje de la violencia: enfoque de derecho, enfoque de desarrollo humano, enfoque de género y enfoque de ciudadanía y cultura de paz, es decir, desde un enfoque integral.

La descripción inductiva, con miras a generar hipótesis o planteamientos teóricos, es un proceso heurístico o de descubrimiento, que permite analizar e interpretar los datos (Sarabia, 1999); por lo tanto, no establece una "generalización estadística", sino una "generalización analítica, es decir, representar o generalizar una teoría." El investigador, o los investigadores, en este caso deben adoptar el papel de "recolectores de datos" (Shaw, 1999), lo cual permite acercarse al fenómeno y ser capaz de descubrir, interpretar y comprender la perspectiva de los participantes de la realidad.

\section{Población y muestra}

El estudio de caso se focalizó en los miembros de dicha comunidad educativa:

- 18 Alumnos de Bachillerato (Con edades entre los 15 y 19 años)

- 12 Padres de Familia (Miembros de la Directiva de Padres de Familia)

- 10 Profesores (Coordinadores y maestros guías de bachillerato)

- Director del Instituto Nacional de Santa Lucía.

El muestreo no probabilístico, criterial o intencional (Russel, 2013) sirvió de mecanismo para poder determinar el grupo de participantes en la investigación como objetos de estudio. Según este procedimiento se seleccionan aquellas muestras que reúnan las características que el investigador ha establecido con anterioridad. Una muestra intencional puede estar influenciada por las preferencias o tendencias, conscientes o inconscientes, de la persona que lo hace.

Se seleccionó al Instituto Nacional de Santa Lucía (INSL) por su ubicación geográfica, su clasificación como uno de los 20 municipios con mayor índice de violencia en el país (FUNDAUNGO, 2012), en el cual se realizó un proceso de "tregua" entre la municipalidad y grupos de jóvenes pandilleriles, y por haber sido declarado como un municipio libre de violencia por las autoridades municipales.

De los alumnos: los alumnos se escogieron de manera aleatoria con el apoyo del director y el coordinador escolar de bachillerato. No se determinó otro criterio más que el hecho de que fuesen estudiantes del INSL, de primero a tercer año de bachillerato técnico vocacional que realizan una jornada de más de 5

Escuela y violencia: estudio de caso del Instituto Nacional de 16. Santa Lucía horas. Los grupos seleccionados fueron dos para poder contrastar respuestas y determinar tendencias desde la opinión de los mismos jóvenes, la participación fue voluntaria, ninguno de los estudiantes se negó a participar. 
De los padres de familia: se eligieron por su representatividad en la institución y el grado de involucramiento en los procesos de la institución educativa, además de coincidir una actividad general de la junta directiva, con la realización del grupo focal con algunos de los padres y madres de la directiva central del instituto. Así se logró tener una representatividad aceptable en el grupo focal para la recolección de los datos. Se contó con la presencia de 10 padres de familia en el grupo focal, del cual 8 eran mujeres.

De los maestros: El perfil que se determinó fueron los maestros guías o coordinadores de algunas secciones del bachillerato, quienes son los que pasan mayoritariamente gran parte de la jornada educativa con los estudiantes, lo que les da la capacidad de conocer a la mayoría de los mismos de sus cursos y de las demás secciones, situación a que los docentes de media jornada u hora clase difícilmente puedan lograr. Además con apoyo del coordinador escolar se buscó a 6 de los profesores con más disponibilidad para realizar el grupo focal, lo que viabilizó la recolección de los datos.

Del director del centro: El director del centro es el responsable directo de la administración y la gestión educativa del INSL. Por medio de su opinión se conoció la visión institucional, la violencia en la convivencia de la comunidad educativa. La accesibilidad y respaldo del director fueron fundamentales para obtener otras fuentes de información.

\section{Técnicas e instrumentos}

La información se recolectó por medio de la técnica de grupos focales aplicada a tres de las cuatro fuentes. Los estudiantes, profesores y los padres de familia, y una entrevista al director del centro educativo. Se determinó utilizar la técnica de los grupos focales debido a su naturaleza heurística, que permite encontrar información más allá de una respuesta cerrada, reacciones, estímulos, posiciones políticas e ideológicas, genera una lluvia de ideas, y lo más determinante investigar cómo la gente interactúa de acuerdo con una situación o realidad determinada (Guest, Namey, \& Mitchell, 2013), que para esta investigación son las concreciones de la violencia en el centro educativo. Los instrumentos fueron aplicados a la comunidad educativa de la siguiente forma:

1. Dos grupos focales de jóvenes, para poder sustentar y contrastar, datos

2. Un grupo focal de docentes

3. Un grupo focal de padres de familia

4. Una entrevista con el director del Instituto

\section{Procesamiento y análisis de los datos}

Todas las sesiones de grupos focales y la entrevista fueron grabadas en audio con el consentimiento de los participantes para ser luego transcritas. Con estos datos se comenzó el proceso de categorización y el análisis mediante la triangulación. Se diseñó una matriz para clasificar los datos de los grupos focales y proceder al vaciado de los datos en la misma para clasificarlos según 
el tipo de violencia que se experimenta en la convivencia del instituto. Luego, se realizo un análisis, es decir, una triangulación de los datos entre sí, pero con un contraste generacional, para sintetizar la información en otra matriz en la cual se determinan tendencias y temáticas sobre las concreciones de la violencia y su relación con la teoría de Galtung.

La triangulación se realizó mediante una segunda matriz en la que la clasificación de las violencias se hizo desde la teoría de Galtung, y se organizaron de acuerdo con tres nuevas categorías que se definieron para consolidar las perspectivas de los participantes de la investigación:

1. Relaciones de poder y figuras de autoridad

2. Igualdad de género

3. Limitantes para el Desarrollo Humano en el aprendizaje

Estas categorías fueron creadas desde las tendencias mediante el análisis de los datos que se encontraron en las manifestaciones de violencia clasificadas en las tablas de vaciado y su respectiva interpretación como símbolos del contexto determinado. Estas tres categorías son inferiores a las de Galtung, pero son necesarias para las primeras, y se establecieron para poder realizar la triangulación y un análisis temático. Estas están determinadas por los patrones, las temáticas y los tipos de violencia que se concretizan en la convivencia de la comunidad educativa.

\section{Resultados}

Los resultados del estudio sobre las manifestaciones de violencia en las relaciones de la comunidad educativa, sobre sus concreciones de la violencia en la comunidad educativa del Instituto Nacional de Santa Lucía (INSL), de acuerdo con sus tendencias se expresan en tres categorías:

\section{Relaciones de poder y figuras de autoridad}

Estas son las relaciones que se dan entre dos personas en una condición desigual, desde el grado de poder que tenga cada una de ellas, lo cual determina las relaciones y la manera de convivir, en donde la parte mejor posicionada en la relación de poder condiciona a la otra.

El patrón cultural de la aceptación de mecanismos de corrección y de enseñanza por parte del profesor a través de acciones violentas sigue siendo la manera de educar en el proceso de enseñanza. Tanto maestros, padres de familia como los mismos estudiantes, manifiestan elementos de una cultura autoritaria que a pesar del proceso de enseñanza aprendizaje no se ha podido prevenir, de manera que la violencia en la corrección sigue siendo el mecanismo para el mejoramiento del rendimiento académico, el mantenimiento del orden y la disciplina.

Escuela y violencia: estudio de caso del Instituto Nacional de

18. Santa Lucía
Esto provoca concreciones de violencia directa por parte de educadores, padres de familia e incluso el estado como el abuso de poder por parte de los cuerpos de seguridad que brindan "protección a la escuela" mediante cateos 
y registros agresivos dentro del instituto de manera arbitraria; los maestros con acciones correctivas por medio de gritos, humillaciones, y los padres con golpes, maltrato psicológico y verbal, que desemboca en una cadena en la que los jóvenes son los últimos en trasladar estos tipos de violencia a los demás, y a su grupo etario en el centro educativo.

La disciplina y el orden de los alumnos están determinados por la condicionante de la presencia o no del educador, es decir, como una manera de control y no como acuerdo de convivencia, hay una dependencia externa del factor de autocontrol y dominio de la conducta individual y colectiva, que desemboca en un doble comportamiento:

"Imagínese una jovencita no la quisieron revisar de los pechos pues! imagínese como lleva un arma allí tan incómodo!". Joven mujer, grupo focal 1.

"Todos los días hacían registro, adentro de los salones afuera, no nos dejaban entrar y cabal cuando vos ibas entrando, y... hubo una ocasión, una sola vez que quitaron los teléfonos y estaban todos los orientadores, tu orientador... pidiendo teléfonos y no podías llamar, no podías... a todos echaron en la colada". Joven, grupo focal 2.

"... de algunos policías bien, y de algunos policías re mal, porque el policía no es bien visto por nuestro estudiante precisamente por el maltrato que hacen fuera de aquí. Yo he visto como maltratan a los estudiantes, los golpean. Maestro "porque yo siento que la violencia más dura es la de tus padres hacia vos, que la de tus compañeros hacia vos, cuando es tus compañeros no te importa, porque que te va importar si ellos no viven con vos". Alumno, grupo focal 2.

"Que le vamos a hacer si los jóvenes ya vienen así desde su familia". Maestro.

"Pero la forma en la que registra. Inclusive yo me hice bien amigo de una policía que había ahí, que era pésima. Es más un día a mí me reviso en la calle, me pararon, y no es mentira, así me agarro los testículos, yo le agarre la mano y se la quité. Y así agarran a los estudiantes, entonces eso se llama maltrato, pero los policías así están fabricados para tratar bestias, nosotros no somos bestias, es un pésimo maltrato de los policías". Maestro.

Esto evidencia que no hay una corresponsabilidad para con el instituto por parte de la familia en el proceso de enseñanza aprendizaje; es decir, que algunos de los jóvenes no reciben los valores fundamentales desde la familia para poderlos desarrollar desde el sistema educativo, ante lo cual el maestro no puede incidir de una manera eficaz. Para los estudiantes la violencia por parte de la familia es peor que la sufrida por los compañeros. 


\section{Igualdad de género}

Esta tendencia se refiere a todas las concreciones de violencia que tienen que ver con aquellas que inciden en la integridad de la mujer, o los comportamientos culturales que legitiman un proceder contra la condición de ser mujer.

Del mismo modo que los aspectos culturales de una sociedad autoritaria están presentes en la convivencia del INSL, el machismo y la cosificación de la mujer siguen siendo patrones de conducta marcados en el imaginario cultural de la comunidad educativa, legitimado por concepciones desde un tipo de violencia cultural.

La mujer sigue siendo cosificada como pertenencia del varón, estigma plenamente aceptado por las alumnas quienes en las relaciones sentimentales con los jóvenes buscan protección y seguridad. Esto provoca "peleas" entre las jóvenes por "ser de alguien" como concreción de violencia instrumental para ganar respeto y ejercer intimidación a los demás.

"aquí ser mujer es ser mujer de alguien". Alumnos, grupo focal 2.

"Es lo que hacen todas las bichas cuando vienen, ser mujer de alguien, es bien... te sentís bien, como nadie te puede tocar".

"si grandes malcriados que son, hay tan tirando besos a uno y no les importa que estén las cámaras de la dirección, tan va de decir ondas de: hay mamacita y otras vulgaridades". Joven mujer, grupo focal 1

Los estereotipos de los roles de hombre y mujer condicionan más al hombre para participar en hechos violentos o delictivos que a las mujeres, a pesar de que una de las jóvenes participantes del grupo focal dos comentó:

"a mí me llevaron jalada 72 horas, porque no pude llamar a mi mamá para que me fuera a recoger cuando el policía me agarró por un relajo en el que nos confundieron". Joven mujer, grupo focal 2.

La violencia ejercida de manera estructural por parte de los agentes de los cuerpos de seguridad, se manifiesta en un acoso verbal a las jóvenes estudiantes por medio de piropos, insinuaciones, comentarios con un sesgo sexual. Lo cual es antagónico con iniciativas de prevención por parte de la Policía Nacional Civil (PNC) sobre la temática:

"... algunos programas que han venido a dar unos policías son sobre violencia de género, eso sí que yo he estado en esas, pero es un aula y dan una charlita". Maestro.

Escuela y violencia: estudio de caso del Instituto Nacional de

20. Santa Lucía
"Una vez estábamos en clases con una compañera y... de repente vamos viendo que nos dice un policía iey bichas!... nos volteaos a ver para atrás, no será a nosotros dije yo, pero es que cabal nos empezó a manosear y 
todo porque nos manoseó y nos empezó a preguntar, nos quitó el teléfono empezó a registrarlos, saco unos números". Joven mujer, grupo focal 2. "iimagínese una jovencita no la quisieron revisar de los pecho pues! ilmagínese como lleva un arma allí tan incómodo!" Joven mujer, grupo focal 1.

Lo que evidencia una clara contradicción debido a la violación del marco legal de protección para la mujer por parte de algunos elementos de la PNC, por medio de la violencia estructural.

La agentes femeninas les registran de una manera sumamente agresiva y poco delicada, incluso con cierta morbosidad en partes íntimas del cuerpo en donde es muy poco probable llevar algún arma; por ello, muchas jóvenes también se sienten acosadas de manera sexual por parte de agentes mujeres.

"Pero también las mujeres policías son bien groseras porque cuando registran allí, casi lo desvisten a uno le tocan, hasta la falda, todo le tocan". Alumno, grupo focal 1.

"He visto mujeres PNC cuando revisan a las señoritas que las tocan demás, eso es bien feo". Maestro.

La igualdad y equidad de género es comprendida de una manera muy sesgada respecto al varón, la igualdad está determinada por el trato a la mujer como si fuese un hombre, es decir, si es capaz o no de cumplir tareas de exclusividad masculina como por ejemplo levantar objetos muy pesados en el taller, arreglar las cosas como los varones:

"A las niñas las tratan igual en el taller solo porque vos vas a ser niña no vas a hacer la práctica, si vos agarraste auto es porque te gustó auto y vas a hacer lo que vas a hacer, no importa que seas niña, o sea se te va a quebrar una uña (jajaj vale verga) pero lo vas a hacer porque lo vas a hacer, siempre esta esa igualdad". Alumno, grupo focal 1.

"yo en mi caso creo que a los varones los quieren más, solo hay dos en el grado, solo se le acercan a la profesora, y ya es diferente con ellos, con nosotras es diferente, gacha. Grupo focal 1.

A pesar de que existe un rico y variado marco legal que protege a la mujer, y que determina los mecanismos para que las diferentes instituciones transversalicen la equidad de género, todos los miembros de la comunidad académica manifestaron desconocimiento de la misma, lo que denota que en el proceso educativo no se está previniendo la violencia contra la mujer.

Lo anterior demuestra que aunque existen las herramientas y mecanismos de igualdad de género, la comunidad académica aún no asume dentro del proceso educativo, la gestión para poder cambiar este tipo de violencia cultural, 
que desemboca en concreciones de violencia institucional y directa como se ha podido apreciar.

\section{Limitaciones al desarrollo humano en el aprendizaje}

Las limitantes para el desarrollo humano en el aprendizaje son los aspectos más relevantes que minan el desarrollo personal de los miembros de la comunidad educativa, y les restringe su derecho a la ciudadanía y a la ciudad.

De acuerdo con los resultados del presente estudio, la dimensión de ciudadanía no es comprendida ni conocida por los jóvenes estudiantes y no poseen claridad de lo que son sus derechos y deberes ciudadanos. Esto implica una erosión en el sentido de bien común y solidaridad que favorece una cultura de anomia; es decir, una desviación, desvinculación o ruptura de las normas sociales, éticas y morales para una sana convivencia social.

El paradigma exclusivo de prevención desde el enfoque de seguridad provoca cierta desnaturalización de las instituciones. Por ejemplo, registros realizados por la PNC en las aulas sin contar con una orden de allanamiento o los requisitos establecidos en los artículos 195 y 196 del Código Procesal Penal que pueden llegar a constituir vulneraciones a los derechos de los alumnos en su integridad física y psicológica, así como también injerencias arbitrarias a su privacidad al revisarles y decomisarles artículos personales, por ejemplo, su celular.

La discriminación por rostro o apariencia es una concreción clara de violencia cultural, por la cual se condena estudiantes, y hasta profesores, por su aspecto físico o su manera de vestir, o ser estudiante del INSL para que a juicio de los cuerpos de seguridad sea catalogado como "sospechoso", y tratado como un delincuente.

"es cierto que el sistema es inclusivo y de oportunidades, pero dentro de esas exclusividades y oportunidades tienen que haber prioridad, si no la hay no estamos colaborando con la sociedad estamos aportando bachilleres que no van aportar nada, y vuelven después de haber terminado vuelven a lo mismo al problema de las pandillas". Director.

"la mujer moderna con la tecnología moderna podía desempeñar cualquier trabajo, pero la mujer moderna con la tecnología antigua, no puede trabajar en las áreas de nosotros los técnicos". Maestro.

Lo anterior evidencia lo complejo que sería darle forma a un modelo de Policía Comunitaria más afín a este tipo de desempeño policial, debido al alto grado de resentimiento y desconfianza por parte de la comunidad académica hacia la policía, por la violación a los derechos humanos del que son víctimas.

Escuela y violencia: estudio de caso del Instituto Nacional de

22. Santa Lucía

A pesar de que el PLANPREVES le asigna responsabilidades a los docentes, de seguridad y prevención, los maestros tienen poca preparación pedagógica y técnica para enfrentar un nuevo escenario en el proceso educativo determinado 
por la realidad que les toca enfrentar. Las intervenciones del Ministerio de Educación no son sistematizadas ni organizadas, consideran que lo que ha funcionado para ellos, es la experiencia en el hecho de acertar o equivocarse en las intervenciones cotidianas en el proceso de enseñanza aprendizaje, más que por los planes o iniciativas de dicho Ministerio, por lo que se hace necesario formar y educar a los maestros y dotarles de las herramientas necesarias para que puedan desarrollar su labor como docentes y educadores y no como agentes del mantenimiento del orden.

\section{Conclusión}

La teoría de Johan Galtung permite comprender la situación de convivencia en la comunidad académica del INSL, ya que las concreciones de violencia directa están íntimamente relacionadas de manera inseparable con las concreciones de violencia estructural y cultural.

Las políticas públicas continúan adoleciendo del mismo mal, o el diseño parte de la concepción de una persona, sin el apoyo de un equipo multidisciplinar, lo que la vuelve una acción populista, o una vez diseñada no hay ninguna vinculación con otra cartera del estado, como el Ministerio de Hacienda, para que pueda inyectársele recursos financieros para territorializarla con eficacia y eficiencia para lograr incidir en el problema que busca solucionar.

Desde lo expuesto por la comunidad educativa del Instituto, podemos constatar que las políticas públicas para intervenir la violencia en las escuelas son abordadas de manera exclusiva desde el enfoque de seguridad. Es decir, de corte tradicional, desde la clasificación de Dina Krauskopf, que se visualiza al joven como un problema que debe resolverse o eliminarse, y no como un sujeto que debe desarrollarse.

La violencia de género es una concreción de violencia cultural, que desata acciones concretas que vulneran o dañan a la mujer en el INSL, de manera que aunque existe un amplio marco legal y herramientas para la igualdad de género, la comunidad educativa debe ser capacitada y habilitada para el uso de técnicas y herramientas que faciliten la inclusión de género al quehacer institucional.

La violencia estructural está determinada por el estado y su política de intervención en las escuelas, que aunque el marco legal le faculta el monopolio de la violencia para ejercer el control y la seguridad. Por lo que las acciones concretas que desde un mal entendido concepto de seguridad pública, terminan volviéndose en limitantes a los derechos constitucionales, a manera de un estado de sitio, lo cual es avalado por la sociedad, como medida de populismo punitivo, que busca un tipo de prevención de choque, por medio de la represión violenta, que termina criminalizando a la juventud en general.

Esta nueva coyuntura requiere políticas nacionales nuevas y también enfoques distintos de las políticas que preparen y acompañen de mejor manera a 
las comunidades educativas, desde una perspectiva interinstitucional para enfrentar los desafíos que los nuevos escenarios plantean al proceso enseñanza aprendizaje. Las intervenciones deben estar diseñadas para poder medir el alcance de mejora.

La prevención de de la violencia instrumental requiere una intervención en las personas de las instituciones educativas con un enfoque preferencial hacia las nuevas generaciones con procesos educativos con una visión de ciudadanía, género y promoción humana, que le ayude a desarrollar sus capacidades, de vivir una vida prolongada y la oportunidad de tener un ingreso decente para vivir.

No solo tenemos derecho a la ciudadanía, sino a la ciudad, el espacio para poder ejercer la ciudadanía por medio de la participación en un contexto de seguridad ciudadana ( $O$ ’Donnel, 201 1), esto podría aplicarse a la educación, ya que los jóvenes no solo tienen derecho a la educación, sino a la escuela (la infraestructura y sus procedimientos) como el lugar seguro donde deba darse sin problemas el proceso enseñanza aprendizaje.

Esta emergencia preventiva debe incidir en los procesos educativos para combatir lo que Matín-Baró definió como la violencia: acción social que se inscribe en una ideología que justifica, legitima y valida el uso de la fuerza contra otros, no como una "visión falsa de la realidad", sino como la visión de la realidad-creencias, valores, usos y costumbres- que cada persona construye en su relación indisoluble con otros individuos en una sociedad determinada. Por ejemplo, un niño que crece en un ambiente violento y es víctima de violencia intrafamiliar, social, adquiere ciertos valores y creencias en cuanto a su rol y la convivencia en el tejido social que pueden derivar en comportamientos antisociales y criminales desde muy temprana edad (Martín-Baró, 2003), por ello el reto de la emergencia educativa actual, nos reta, nos implica a "Llegar a tiempo", como lo propone el Sistema Preventivo Salesiano, si de verdad queremos a mediano y largo plazo empezar a salir de este círculo de violencia, por medio del mejor mecanismo de prevención: La educación.

Escuela y violencia: estudio de caso del Instituto Nacional de

24. Santa Lucía

\section{Referencias}

Calderón, P. C. (2009). Theory of Confl icts by Johan Galtung. Revista Paz y Conflictos, 60-81.

Fisas, A. V. (1998). Cultura de paz y gestión de conflictos. Barcelona: Icaria.

FLACSO. (enero de 2013). Fondo para el logro de los objetivos del desarrollo del milenio (F-ODM). Recuperado el 21 de junio de 2014, de http://www.mdgfund.org/es/ publication/el-sector-educativo-contra-la-violencia-en-el-salvador

FUNDAUNGO (2012). Atlas de la violencia en El Salvador (2005-2012). San Salvador: GRAFIKA.

Galtung, J. (2006). La trasformazione dei conflitti con mezzi pacifici (II Metodo TRANSCEND) United Nations Disaster Management Training Programme. Torino: Centro Studi Sereno Regis. 
Galtung, J. (1998). Tras la violencia. Bilbao: Gernika Gogoratuz.

Galtung, J. (2003). Violencia Cultural. Billbao.

Guest, G., Namey, E., \& MitchellL, M. (2013). Collecting Qualitative Data. Los Angeles: SAGE Publications Inc.

Lemus, E. (19 de AGOSTO de 2010). BBC MUNDO. Recuperado el 01 de julio de 2014, de BBC MUNDO: http://www.bbc.co.uk/mundo/america_latina/2010/08/100819 m_ 1632 el_salvador_violencia_escuelas_estudiantes_jg.shtml

Martín-Baró, I. (2003). Poder, ideología y violencia. Madrid: Trotta.

Martínez, P. C. (2006). El método de estudio de caso Estrategia metodológica de la investigación científica. Pensamiento y Gestión, 166-193.

O'Donnel, G. (2011). Democracia de ciudadanos. New York: PAIDÓs.

PLANPREVES, M. (2013). Plan Nacional de Prevención y Seguridad en las Escuelas (PLANPREVES). San Salvador: MINED.

PNUD, 2. (2014). Informe sobre Desarrollo Humano El Salvador 2013. Imaginar un nuevo país. Hacerlo posible. Diagnóstico y propuesta. San Salvador: Impresos Múltiples.

Russell, B. H. (2013). Social Research Methods: Qualitative and Cuantitative aproaches. California: SAGE.

Sarábia, F. J. (1999). Metodología para la Investigación. Madrid: Pirámide.

Shaw, E. (1999). A guide to a Qualitative Research process: Evidence from a Small Firm Study. Qualitative Market Research: An International Journal, 57-70. 